\title{
Interhemispheric connections between primary visual areas: beyond the midline rule
}

J.-C. Houzel,

M.L. Carvalho and R. Lent
Departamento de Anatomia, Instituto de Ciências Biomédicas, Universidade Federal do Rio de Janeiro, Rio de Janeiro, RJ, Brasil

\author{
Correspondence \\ J.-C. Houzel \\ Laboratório de Neuroplasticidade \\ Departamento de Anatomia \\ ICB, CCS/F31, UFRJ \\ 21941-590 Rio de Janeiro, RJ \\ Brasil \\ Fax: +55-21-2561-7973 \\ E-mail: jchouzel@anato.ufrj.br \\ Web: www.anato.ufrj.br/jchouzel/lab \\ Presented at the IV International \\ UNESCO Course on "What the \\ Developing Cerebral Cortex Tells \\ About the Adult Cortex (and Vice \\ Versa)", Rio de Janeiro, RJ, Brazil, \\ December 3-7, 2001.
}

Research supported by CNPq (Nos. PV301098/98-0 and APQ477864/01-5), PRONEX (No. 661052/1997-4), and FAPERJ (No. E26/150-110/02).

Received July 12, 2002 Accepted August 20, 2002

\begin{abstract}
In the last five years, a number of detailed anatomical, electrophysiological, optical imaging and simulation studies performed in a variety of non-human species have revealed that the functional organization of callosal connections between primary visual areas is more elaborate than previously thought. Callosal cell bodies and terminals are clustered in columns whose correspondence to features mapped in the visual cortex, such as orientation and ocularity, are starting to be understood. Callosal connections are not restricted to the vertical midline representation nor do they establish merely point-to-point retinotopic correspondences across the hemispheres, as traditionally believed. In addition, anatomical studies have revealed the existence of an ipsilateral component of callosal axons. The aim of this short review is to propose how these new data can be integrated into an updated scheme of the circuits responsible for assembling the primary visual field map.
\end{abstract}

Key words

- Corpus callosum

- Visual cortex

- Perceptual binding

- Vertical midline

- Orientation column

- Binocular interactions

\section{Introduction}

The major fiber bundle in the human brain, the corpus callosum, contains around 200 million axons that link the two cerebral hemispheres. Despite the amount of research devoted to this pathway $(1,2)$ and the great popularity of the concept of lateralization of functions, the neuronal mechanisms of interhemispheric cooperation are largely unknown. The lack of a general working model for the functional organization of transcallosal circuits is probably due to the multiple functions of callosal fibers, since they arise from almost all regions of the neocortex. The few generalizations on the sensory portions of the corpus callosum are based on incom- plete experimental data that are now being complemented in such a way that the main ideas ought to be rediscussed.

In associative areas, interhemispheric connections exist between both heterologous and homologous regions. In primary sensory areas, however, the situation is considered to be simpler since connections are presumed to be essentially homotopic, i.e., they reciprocally link corresponding regions located in symmetric sectors of the hemispheres. Moreover, most callosal connections between primary sensory cortices appear to be restricted to portions of these areas where the "midline" is mapped (reviewed in Ref. 3): in the primary somatosensory cortex, callosal connections are densely packed in regions repre- 
senting the central parts of the trunk, the face, and the proximal segments of the limbs, whereas they have been repeatedly reported to be sparser or virtually absent from the representation of distal segments. Similarly, callosal connections within the primary visual cortex are often believed to be restricted to the representation of the vertical midline of the visual field. Since each hemisphere only receives thalamic input from the contralateral half of the body or the visual field, it is reasonable to assume, as did Ramón y Cajal (4), that one essential function of the corpus callosum is to guarantee the continuity of the sensory maps across the hemispheres. The established view is, therefore, that the fusion of primary cortical representations is achieved through precise, reciprocal, point-to-point callosal connections between cortical neurons whose receptive fields are located along the midline fracture, as in a string of press buttons.

The basic layout of the callosal connections between primary visual areas in adult mammals has been reviewed recently (5). Briefly, classical anatomical and electrophysiological experiments, mostly performed in cats, have demonstrated the following features: a) Callosal connections are concentrated along the border between areas 17 (A17) and 18 (A18). This is not a sharp boundary, but rather a transition zone whose cytoarchitectonic and physiological response properties are intermediate between those of A17 and A18 (6,7). b) Extensive receptive field mapping of the 17/18 border, confirming earlier recordings from the fibers running within the posterior part of the corpus callosum (8), indicates that the callosally connected parts of visual areas include, on each side, the representation of the vertical midline together with a narrow portion of the ipsilateral hemifield (7). c) Recordings from split-chiasm cats, in which the geniculocortical and transcallosal pathways can be differentially activated by visual stimulation of one or the other eye, reveal that ipsi- and contralateral inputs converging onto callosal recipient neurons are closely matched in terms of retinotopic location, size, and orientation selectivity $(9,10)$. d) Taken as a whole, these and other observations suggest that, indeed, callosal projections are functionally equivalent to corticocortical projections, being only longer-reaching $(1,6)$.

During the last few years, however, a number of detailed anatomical, electrophysiological, imaging and simulation studies performed by different research groups in a variety of species have revealed that the functional organization of callosal connections is more elaborate than previously thought. Important pieces of recent information deal with the columnar and retinotopic arrangement of callosal connections. In addition, we shall mention unpublished observations from our laboratory about the ipsilateral component of the interhemispheric integration. The aim of this short review is to propose how these data can be integrated into an updated scheme of the circuits responsible for assembling the primary visual field map.

\section{Relation of callosal patches to other columnar modules}

Early degeneration studies indicated that the density of callosal terminals is not homogeneous across the cortical surface. On a finer scale, callosal terminals and, to a lesser extent, callosal neurons were later reported to form a patchy pattern in many cortical areas of various species, including the 17/18 border zone of the cat (6). In order to decipher the functional logic of interhemispheric projections, an important issue is how these "callosal columns" relate to other modular systems of the visual cortex. Given the presumed analogy between callosal and horizontal connections, together with the matching between thalamic and callosal inputs converging onto target cells (see above), it seemed natural to propose that callosal fi- 
bers connect neurons within columns of similar orientation selectivity. However, recent studies have ruled out this hypothesis.

In 1994 and 1996, two independent studies demonstrated that both callosal neurons and terminals are densely packed within cytochrome oxidase-rich domains of the visual cortex in cats (11) and macaques (12). Although the connectivity associated with this pattern has recently been elucidated in the latter species (13), the functional significance of cytochrome oxidase modules in other species remains unclear. In 1997, Schmidt et al. (14) used a combination of deoxyglucose, optical recording and retrograde tracing in strabismic cats and concluded that callosal projections were preferentially established between columns of the same orientation, as is the case for intrahemispheric long-range projections. However, although converging thalamic and callosal inputs still appear to be matched for orientation in strabismic cats (15), it is known that the overall distribution of callosal projections and terminals is profoundly modified (16). Therefore, the relation of the pattern of callosal connections to the columnar systems of the visual cortex in normal adults cannot be inferred from data obtained in animals with altered visual experience. Indeed, contrasting results were obtained by Bosking et al. (17) who achieved optical imaging of both orientation preference and retinotopic position maps in normally reared tree shrews. These authors concluded that callosal connections lack the orientation specificity characteristic of long-range horizontal connections (18), but "are arranged in a topographic fashion that links visuotopically corresponding sites in the two hemispheres". If this turned out to be the case in other species, callosal fibers would be more likely to serve a function comparable to that of local intrinsic networks, preserving the continuity of the visual field map across the hemispheres. Finally, Olavarria $(19,20) \mathrm{dem}-$ onstrated that the callosal mapping relates neither to orientation specificity, nor simply to retinotopy, but rather to areal location and ocular dominance. The new results only appeared after he made a careful distinction between the 17/18 border and the flanking parts of A17 and A18 themselves, and can be summarized as follows: callosal neurons located within the transition zone, where a narrow portion of the ipsilateral visual field is mapped, preferentially occupy contralateral ocular dominance columns and project to A17 and A18 of the opposite hemisphere. In contrast, callosal neurons located slightly off the vertical midline representation - i.e., within the lateral-most part of A17 and the medial-most part of A18 - occupy ipsilateral ocular dominance columns and converge onto the opposite transition zone. This finding, compatible with another recent report (21), has important implications for understanding how callosal connections are selected throughout development (see Ref. 2). In addition, it calls for a reinterpretation of the circuits presumed to be responsible for the unification of the two hemi-representations of the visual field.

Yet, Olavarria's observations were based on tangential plots of populations of cells retrogradely labeled after fairly large tracer injections. Therefore, they might not have disclosed the complete connectivity scheme, which must be even more intricate. Threedimensional reconstructions of biocytinlabeled individual callosal axons from the cat visual cortex revealed that they often terminate into distinct radial columns of the target hemisphere $(5,22)$. For most axons, the heaviest terminal columns are located along the 17/18 border, while additional branches with sparser boutons arborize within A17 and/or A18. It is difficult to assess whether such diverging terminal branches would have incorporated the different tracers used in Olavarria's experiment, since his injections were intentionally aimed at one or the other cortical region. Actually, although double- or triple-labeled cells do not seem to 
have been investigated systematically, a few instances of overlap between populations of callosal cells labeled by injections placed at the 17/18 border and A17 can be detected (see Figure 1 in Ref. 20).

In any case, it is important to emphasize that many features (such as orientation, ocularity, and spatial frequency) are mapped onto the surface of the visual cortex, each modular pattern being superimposed on the retinotopic map, resulting in a multidimensional layout that seemingly optimizes the uniform coverage of each feature for each point of the visual field (23). Therefore, callosal neurons within an ocular dominance column also display their own orientation selectivity and the recent findings do not contradict electrophysiological evidence of matched orientation between thalamic and callosal inputs both in normal and strabismic cats (15).

\section{Functional implications of the topographic divergence of callosal connections}

A second critical issue is the topographic precision of callosal connections. Recordings from split-chiasm cats indicated, in addition to matched orientation between thalamic and callosal afferents, a precise correspondence of retinotopic position, as evidenced by the high degree of receptive field overlap, which increases progressively with normal binocular experience (10). This was compatible with the classic view that callosal connections perform a point-to-point topographic mapping between corresponding loci located in symmetric regions of the two hemispheres, and thereby contribute to the construction of receptive fields in the vicinity of the vertical midline.

Studies aimed at visualizing the overall distribution of callosal projections using very large injections of retrograde and anterograde tracers, or based on the patterns of degeneration staining after section of the corpus callosum, have revealed that the distribution of callosal neurons is broader than that of callosal terminals $(11,19,24,25)$. Therefore, one might assume a certain degree of convergence onto the 17/18 border. On the other hand, the fact that injections of retrograde tracers in A17 or in A18 label cells at the contralateral $17 / 18$ border (20) suggests a possibility of divergent projections. Provided that the degree of anatomical convergence and divergence is reasonably small, these findings should not contradict the electrophysiological data since, especially within regions of highly magnified representation, the retinotopic distance between origin and target neurons would not exceed the angular distance between their receptive fields. However, by combining optical mapping of retinotopic position and retrograde labeling in tree shrews, Bosking et al. (17) found that, although callosal connections are highly topographical, "it is also clear [...] that these connections provide input from a moderate-sized region surrounding exact correspondence. Thus, a particular site in one hemisphere would receive inputs via callosal connections primarily from sites in the other hemisphere that had overlapping receptive fields, but also from sites whose receptive fields could be displaced by as much as the receptive field diameter of a layer $2 / 3$ neuron $\left(5^{\circ}\right)$ ". Even though optical imaging data obtained for intact tree shrews cannot be compared with receptive fields plotted from split-chiasm cats, they strongly suggest that many neurons receive callosal inputs that are not congruent with their thalamic input and therefore do not directly contribute to the generation of minimum response fields.

This assumption is supported by the examination of single callosal axons which, as mentioned above, frequently distribute some conspicuous terminal branches within A17 and/or A18, in addition to densely ramified terminals packed along the 17/18 border $(5,22)$. Secondary terminal columns could 
be located as far as $2500 \mu \mathrm{m}$ away from the $17 / 18$ border. Although we did not attempt to map the visual field representation before processing the brains for serial reconstruction of single axons, retinotopy can be roughly estimated on the basis of extensive receptive field mappings of the lateral gyrus $(10,24)$ : at the same rostrocaudal level, a displacement of $2500 \mu \mathrm{m}$ on the cortical surface away from the $17 / 18$ border would correspond to at least $15^{\circ}$ of eccentricity within the contralateral hemifield, for an elevation of about $-5^{\circ}$ (Figure 1). Thus, far-reaching callosal terminals are likely to contact neurons whose receptive fields are not included within the portion of the visual field transferred through the corpus callosum. On the other hand, recordings from split-chiasm cats indicated that, with very few exceptions (26), transcallosally driven unitary responses elicited by visual stimulation are virtually absent outside the 17/18 border.

\section{Possible role of callosal inputs near the vertical midline representation}

The discrepancy between the distribution of axonal terminals and physiological responses emphasizes the issue of the function of the heterotopic callosal projections terminating at the flanks of the $17 / 18$ border. Since most callosal neurons are binocular, splitting the chiasm suppresses half of the thalamic afferents that might drive callosal neurons in intact animals. Therefore, one cannot exclude the possibility that the absence of transcallosal responses outside the $17 / 18$ border is an artifact due to the dampening of normally suprathreshold inputs from a subpopulation of callosal cells whose firing would be particularly dependent on binocular stimulation. Unfortunately, connectivity studies using electrical stimulation, which might have disclosed more subtle inputs, explored mainly the 17/18 border $(27,28)$. A simpler assumption is that, for any given callosal axon, dense terminal col- umns provide massive synaptic inputs and trigger spiking responses of the target neurons, whereas secondary terminal columns with sparser boutons form weaker contacts and provide only subthreshold activation.

Given that secondary columns frequently occur in non-symmetric locations with respect to the cell body of the parent neuron, this arrangement could endow interhemispheric networks with an interesting property of anticipation. Let us consider a common situation where, as we keep a steady frontal gaze, a car appears on the left and crosses the entire visual field, from left to right (Figure 2). As a result of the topography of the retinogeniculocortical pathway (for the sake of simplicity, we will restrict the example to a feline-like A18), the visually driven activity in the cortex will first

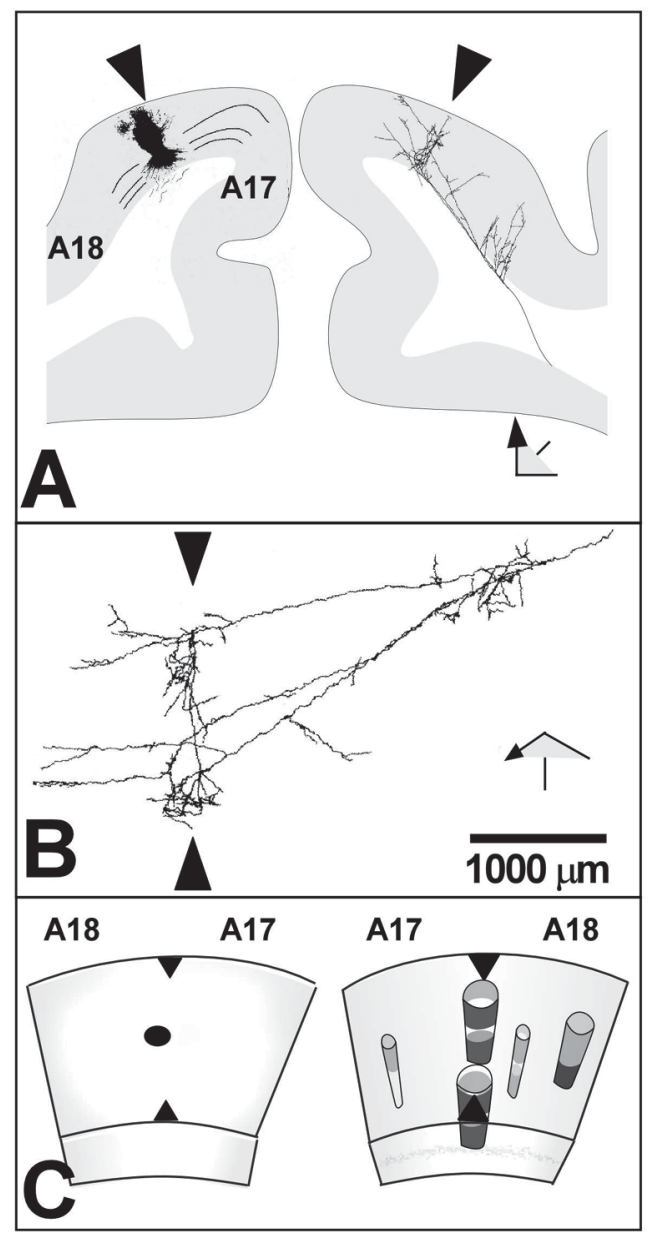

Figure 1. Callosal axon with a divergent termination pattern in the cat visual cortex. Threedimensional reconstruction of a callosal axon labeled by extracellular injection of biocytin within the 17/18 transition zone in the cat. $A$, Coronal view of the injection site (left hemisphere) and contralateral arborization of a single callosal axon (right hemisphere). Arrowheads indicate the center of the transition zone between areas 17 and 18 (A17, A18). B, Enlarged surface view of the terminal arborization, obtained after rotation of the three-dimensionally reconstructed axon and correction for tissue shrinkage. Arrowheads indicate the center of the transition zone between A17 and A18. C, Schematic diagram illustrating the topographic relation between the injection site (left) and the terminal territories (right) of the axon shown in $A$ and $B$. Contralateral terminal arborizations are arranged in two main radial columns positioned along the 17/18 border within the representation of the vertical midline - and three minor columns located in A17 and in A18. One can infer from retinotopic maps that the location of the most lateral column corresponds to the representation of about 15 degrees of eccentricity (modified from Ref. 5). 
appear in lateral parts of the right A18, and then gradually shift towards more medial loci as the moving object gets closer to the center. As the car proceeds across the midline and then towards the right periphery, cortical activity shifts to the left hemisphere, starting medially at the 17/18 border and then assuming successively more lateral loci. In a model with purely homotopic, point-topoint, callosal connections (Figure 2B), cortical activity is suddenly transferred from

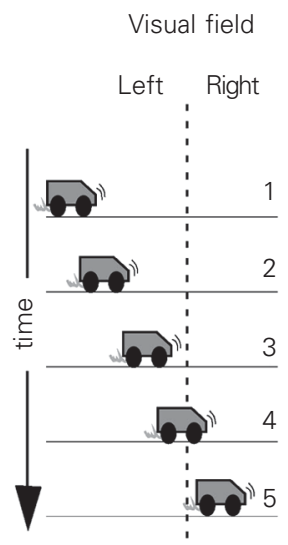

A

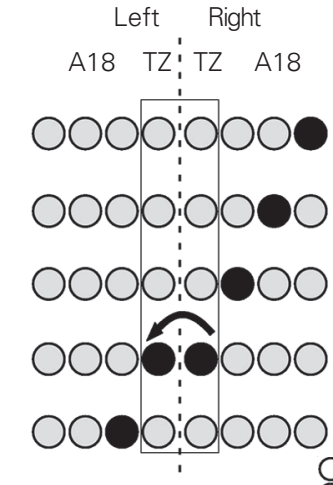

B
Cortical maps

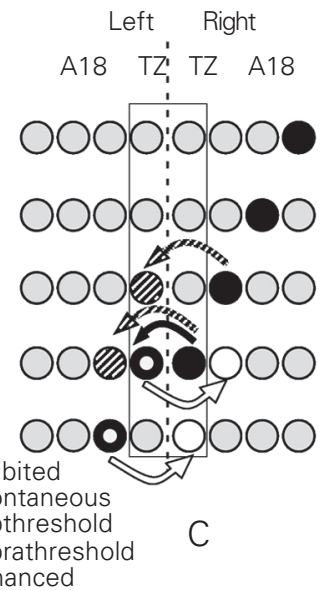

Figure 2. Subthreshold heterotopic callosal inputs could pre-activate selected cortical neurons. A, Visual field. As the observer keeps a steady frontal gaze, a moving car crosses the visual field from left to right. The dotted line indicates the position of the vertical midline of the visual field (central vertical meridian). Numbers 1 to 5 indicate the position of the car at successive time points. $B$ and $C$, Schematic maps of visual cortical area 18 (A18) and of the 17/18 transition zone (TZ) of the left and right hemispheres. The vertical dotted line separates the two sides of the brain. Callosal neurons and terminals are densely packed at the transition zone (box), where the vertical midline is topographically represented once in each hemisphere. Discs represent neighboring cortical loci, which are shaded according to their state of activation, from the inhibited (white) to the basal level of activation (light gray) to subthreshold excitation (dark stipple) to firing (black) and to correlated firing (black with white center spot). Successive horizontal rows correspond to time points 1-5. B, As the car proceeds from left to right, visually driven cortical activity (black discs) shifts from lateral to medial loci in the right $A 18$, and then from medial to more lateral loci within the left hemisphere. With exclusively homotopic connections (black arrow) between the two TZ, cortical activity is suddenly transferred from the right to the left hemisphere at the moment when the moving object reaches the midline (time point 4). C, Heterotopic connections providing subthreshold inputs are added. At time point 3 , inputs converging from the right A18 onto the left TZ enhance the excitability of the latter region before the object actually reaches the midline, in a feature-selective manner (see text for details). At time point 4 , visually evoked responses at the left TZ are already correlated ("enhanced"), which could speed up their further joint processing. At the same time point, subthreshold inputs diverging from right TZ onto the left A18 extend the pre-selection process to regions flanking the vertical midline representation. Inhibitory components (white arrows and discs) are also included, which may permit the subsequent restraining of the network activity. one hemisphere to the other at the time when the object reaches the midline. According to the classic view, the absence of a perceptual break could be attributed to the duplicate representation of the central visual field and to continuity-providing connections between the two maps. Additionally, one can now insert reciprocal, non-mirror symmetric, heterotopic projections providing weak inputs to the flank of A18 and the 17/18 border (dashed and white arrows in Figure 2C). In this case, subthreshold activation of $17 / 18$ border sites driven by callosal neurons in the right A18 would be initiated before the visual stimulus actually reaches the midline, which might speed up further processing. Similarly, at the next time point, callosal neurons located at the right $17 / 18$ border would provide subthreshold activation of left A18 recipient neurons, and prepare the local network for processing incoming thalamic inputs.

We propose that subthreshold transcallosal inputs influence ongoing activity in a topographically organized manner, and thereby enable the pre-activation of selected neuronal populations within the hemisphere that is not yet receiving any input through the retinogeniculate pathway but that, with a very high probability, will do so as soon as the incoming stimulus reaches the vertical midline. This anticipatory mechanism would permit faster processing of stimulus features that need to be integrated across the visual field.

The scheme can be expanded with connections to and from A17, and might also include inhibitory components which, either directly through inhibitory callosal neurons (29) or indirectly via local interneurons (30), could ease the returning of previously activated cortical loci to the basal level. Clearly, this hypothesis does not involve solely callosally connected neurons, but rather relies on the participation of local intrinsic networks within each of the two hemispheres. It also provides a framework for top-down 
influences from higher-order areas to dynamically modulate the speed and scope of interhemispheric integration, according to spatiotemporal patterns of subthreshold activity that would reflect the architecture of callosal connections.

Several lines of evidence support this hypothesis: a) Ongoing spontaneous activity, which is largely responsible for the great variability of evoked neuronal responses, is not random but rather reflects the functional architecture of the visual cortex (31). Spiketriggered averaging of images from the cortical surface, obtained with voltage-sensitive dyes, indicates that the fluctuations in excitability are correlated across columns of similar orientation (32). b) These spatially organized spontaneous fluctuations of the basal level of activity affect the latency of neuronal responses to visual stimuli and enhance their correlation in a state-dependent and feature-selective manner (33). c) Precisely correlated responses are more likely to be grouped for further joint processing, since synchronized inputs have a stronger impact on high-order target neurons than temporally uncorrelated spikes (reviewed in Refs. 34,35). d) As is the case for long-range horizontal connections, callosal connections are selected during development according to experience-dependent rules $(6,15,36)$. Accordingly, their architecture is presumed to reflect frequently occurring constellations of features $(37,38)$. e) Although individual callosal axons display variable architectures in adults, in most cases the geometrical properties of their different branches seem to equalize the conduction delay to all their terminal columns $(5,39)$. Consequently, callosal axons are likely to transmit synchronous inputs to widely separated territories within the target hemisphere. Such inputs would elicit synchronous firing of postsynaptic target neurons within regions receiving dense callosal terminals, as well as exert temporally correlated subthreshold influences within selected territories innervated by less compact terminals. These correlated modifications in membrane excitability would allow a precise synchronization of the very first spikes of the responses subsequently evoked by the appearance of the visual stimulus in the receptive fields of pre-selected neurons (33). Actually, psychophysical evidence indicates that such a grouping process requires no more than a few tens of milliseconds (40). Topographically divergent callosal connections, even though they apparently do not participate in the construction of visual receptive fields, could make a critical contribution to speeding up perceptual binding.

Complementary roles for the widespread, subthreshold callosal inputs can be considered. A recent electrophysiological study (41) indicates that, after binocular vision is impaired for a few weeks in the adult cat by monocular deprivation or ocular deviation, transcallosal receptive fields lose their orientation specificity and increase in size. Although local modulation of inhibitory mechanisms or genuine sprouting of callosal arbors cannot be excluded, the authors suggest that this plasticity is due to strengthening of previously weak synaptic inputs. Along the same lines, subthreshold callosal inputs could also provide a substrate for faster changes, such as dynamic contextual modulation of neuronal responses (42).

\section{Callosal connections within the representation of the peripheral visual field}

Callosal connections between primary visual areas that manifestly violate the vertical midline rule are observed in rodents. In addition to dense callosal connections packed along the 17/18a border, the entire mediolateral extent of rat striate cortex contains numerous callosal cells and terminals, forming a continuous band in infragranular layers (43). Further experiments based on targeted injections of retrograde tracers suggested a dual connectivity scheme (44): a) at the 17/ 
18a border, non-mirror symmetric reciprocal connections would link cortical loci with roughly matching retinotopic projections along the vertical midline representation, as in cats; b) within A17, in contrast, callosal connections would link mirror-symmetric cortical regions, representing mirror-symmetric positions in the periphery of the visual field.

Using sensitive anterograde tracers and computer-assisted microscopy, we confirmed that the proposed connectivity scheme holds

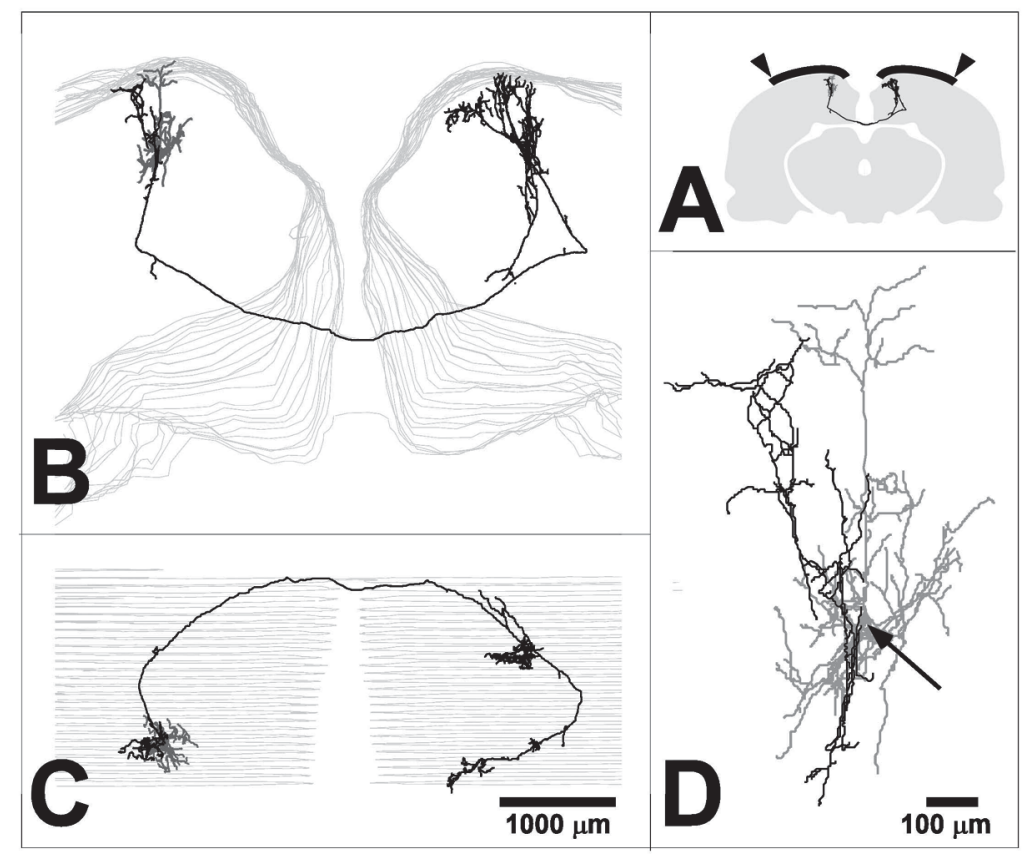

Figure 3. Callosal neuron within the representation of the peripheral visual field in rat A17. Three-dimensional reconstruction of a callosal neuron labeled by a small extracellular injection of biocytin within the medial-most part of the left A17 in a rat. $A$, Outlines of a coronal section of the rat brain, indicating on both sides the mediolateral extent of the cytoarchitectonically defined A17 (thick black line) and the location of the vertical midline representation (arrowheads) inferred from published retinotopic maps. This neuron connects mirror-symmetric cortical loci representing the far periphery of the right hemifield (cell body in the left hemisphere) and the far periphery of the left hemifield (terminal columns in the right hemisphere). $B$, Enlarged frontal view of the whole neuron (gray: cell body and dendritic tree; black: axon), as reconstructed from 29 consecutive, 70- $\mu \mathrm{m}$ thick coronal sections, whose outlines are delineated by the thin light lines. In the left hemisphere, an ascending axonal collateral forms a terminal arbor within the column containing the cell body. Same scale as in C. C, Dorsal view obtained after 90-degree rotation of the threedimensionally reconstructed neuron along the mediolateral axis and correction for tissue shrinkage. In the left hemisphere, the degree of overlap between the dendritic and ipsilateral axonal arbor can be appreciated. In the right hemisphere, two regions of dense branching can be seen at different rostrocaudal levels. Rostral is at the top. D, Enlarged coronal view showing the cell body (arrow), the dendritic arbor (gray) and the ascending ipsilateral axonal collateral. at the level of individual neurons. Figure 3 shows one callosal neuron labeled by an injection of biocytin in the medial-most region of the left A17, where the extreme periphery of the right visual hemifield is mapped. The axon travels rostrally to reach the corpus callosum (Figure 3C), crosses the midline, and then proceeds caudally to reach a fairly symmetric location in the right hemisphere, where the extreme periphery of the left visual field is mapped. On its way, the axon also sends an ascending collateral that forms a very conspicuous terminal column, about $1000 \mu \mathrm{m}$ more caudal than the other one.

Although it is not known whether mirrorsymmetric projections provide supra- or subthreshold inputs to target neurons, they are likely to be involved in processing requiring large-scale integration of features across the entire visual field. They could participate in the computation of optic flow and other visual cues related to locomotion (45) or, likewise, in the detection of symmetric shapes, which is behaviorally relevant not only for highly specialized functions such as face recognition in humans, but also for a variety of tasks in different species (46).

Similar mirror-symmetric patterns of interhemispheric connections have been demonstrated or suggested in extrastriate areas of a number of species, such as areas 20a and PMLS of cats (47) and area MT of macaques and marmoset monkeys (48). Given the small number of differentiated visual areas in rodents, one may speculate that their less specialized primary visual area is responsible for additional processing that is implemented by supplementary areas committed to specific operations in higher mammals.

\section{Ipsilateral axonal collaterals and interhemispheric cooperation}

So far, we have only addressed issues regarding the organization and the function of the signals transferred from one hemi- 
sphere to the other. However, several lines of evidence suggest that callosal neurons also provide ipsilateral inputs through axonal collaterals arborizing within the hemisphere of the parent cell body (Figure 3D). The use of multiple fluorescent retrograde tracers revealed that: a) callosal neurons survive the transection of their callosal axon (49); b) a few extrastriate neurons projecting to the contralateral $17 / 18$ border also contact the homologous ipsilateral region (50), and c) during development, many primary visual cortical neurons with a transient callosal projection end up by establishing contact within the ipsilateral hemisphere (51). These findings are compatible with the ultrastructural observation of synapses labeled in the hemisphere contralateral to an injection of retrogradely transported peroxidase (29), and with occasional drawings of peroxidase-filled callosal neurons showing proximal axonal branches possibly directed to the ipsilateral hemisphere in cats (52). Taken as a whole, these findings are compatible with the possibility that callosal neurons in A17 of adult mammals send ipsilateral axonal collaterals.

To specifically address this issue, we injected very small amounts of biocytin or biotinylated dextran amine into the rat striate cortex. With the help of a computer-coupled light microscope (Neurolucida, MicroBrightfield Inc., Colchester, VT, USA), selected callosal neurons were then reconstructed in three dimensions from consecutive coronal sections, including their entire axonal tree as well as their dendritic arbors. The neuron shown in Figure 3 was labeled by an injection into medial A17. In addition to the contralateral axonal arbor described above, it also gives off a short-range recurrent branch, ascending radially shortly after the axon hillock, and arborizing locally within the column of the parent cell body. Other callosal neurons, located along the vertical midline representation at the $17 / 18$ a border, display more widespread ipsilateral branches. These can also be seen to stem from proxi- mal segments of the axonal trunk but then run parallel to the cortical surface and terminate at variable distances away from the cell body or, alternatively, to arise from more distal portions of the axon, travel within the subcortical white matter and then make a sharp U-turn to re-enter the cortex. The topographic relationships between short- and long-range ipsilateral projections and their contralateral counterparts are currently being analyzed.

Since individual neurons with bi-hemispheric collaterals provide inputs to both hemispheres, they are presumably pivotal players in the interhemispheric integration of the visual scene. One current hypothesis is that the grouping of object features is achieved through the selection of neuronal responses on the basis of their temporal correlation (see above). Stimulus-dependent, millisecond precision synchronization of interhemispheric responses was shown to require the integrity of the corpus callosum $(53,54)$. Although such precise coupling could theoretically be accomplished through reciprocal connections or local oscillating circuits, the simplest and fastest way to produce zero-phase lag synchronization between remote groups of neurons is through common input. Callosal neurons with bi-hemispheric axons appear to be unique candidates to supply such inputs.

On the basis of computer simulation, we have suggested previously that the architecture of callosal axons seems very well suited to promote the synchronous activation of spatially dispersed terminal columns within the target hemisphere. Adjustments of the caliber of axonal branches feeding distinct columns seem to compensate for their differential length, which would allow propagating action potentials to invade distant terminal clusters almost simultaneously (see above and Refs. 5,39). If a similar tuning of the geometrical properties of ipsilateral branches was to be achieved during development, the architecture of bi-hemispheric callosal neu- 
rons would meet the criteria required for providing common, synchronized inputs to both sides of the brain.

As discussed above, it is not critical that the inputs be strong enough to evoke the firing of target cells. Temporally correlated subthreshold influences could modulate membrane excitability in such a way that subsequent, stimulus-evoked discharges would occur in synchrony. More important is that elements receiving the synchronizing influence actually represent subpopulations of neurons selected according to criteria that make some sense for further perceptual processing. Therefore, the previously discussed issue of the relation of "callosal columns" with other modular domains needs to be reexamined in light of the likelihood that postsynaptic targets of callosal neurons in the two hemispheres share some functional properties. For example, it will be interesting to know whether ipsilateral terminals of callosal axons are also located in cytochrome oxidase-rich domains, since both contralateral terminals and callosal cell bodies were shown to be (11). Along the same lines, determining whether ipsilateral terminals of $17 / 18$ border callosal neurons are located in the same or different ocular dominance columns than ipsilateral terminals of A17 or A18 callosal neurons, and whether this eventual bias corresponds or not to the bias observed for contralateral terminals (see above and Ref. 20), might help to understand the functional logic of interhemispheric circuits. In this respect, further investigations of the functional topography of callosal connections will require approaches that allow the analysis of the patterns of afferent and efferent projections in both hemispheres. Threedimensional, whole-neuron reconstruction allows direct analysis of topographic relations between local and remote targets of individual neurons, which makes it a powerful, although time-consuming, approach. Many of the questions discussed above could be answered through experiments combin- ing reconstruction with multiple labeling, optical imaging and multiple electrode recordings in the same animal.

\section{Conclusions}

Rather than contradicting the classical midline fusion hypothesis, the recent observations summarized here give new hints about how interhemispheric integration could actually be achieved.

Callosal connections share some of the properties of local intrinsic connections: by preserving neighborhood relationships, they do contribute to the generation of receptive fields close to the vertical midline. However, they do also connect neurons with non-congruent receptive fields. On the other hand, callosal projections share important traits with long-range horizontal connections (55): they are selected according to correlation rules during development, and mediate precise temporal coupling between distant territories in adults. But they do not exclusively link domains of matching orientation specificity. Interhemispheric connections between the primary visual maps thus establish an elaborate set of relationships among multiple elementary features of the visual scene (orientation, retinotopy, ocularity).

A more comprehensive view of the role of interhemispheric connections in the integrated processing of sensory information might emerge from the study of other species and systems. Recently, physiological and anatomical data were obtained for the ferret $(56,57)$, a model which should help a) clarify the issue of ocular $v s$ retinotopic specificity of callosal connections, since in this species the primary cortical representation of the central visual field is monocular (58), and b) investigate early developmental events, since ferrets are born at an earlier stage of neurogenesis than other carnivores. For instance, what are the developmental constraints and mechanisms governing the selection of appropriate connections in either hemisphere? 
Along the same lines, the recent description of the layout of visual commissural connections in a marsupial (Didelphis aurita, Ref. 59) should add to the evolutionary perspective. In the barrel cortex of the rat, it was recently shown (60) that the stimulation of each vibrissa produces a transcallosal inhibitory "echo" whose spatiotemporal scope can be analyzed in detail due to the precise topography of this system. The somatosensory representation of the body is also highly modular, and is therefore an appealing system. In this respect, large Amazonian rodents provide an unparalleled experimental model since cortical maps can be investigated in great detail across the smooth and large surface of their brain, combining modern physiological and anatomical techniques, such as optical mapping, multiple electrode recordings and computerized morphometry. Preliminary results obtained for the agouti (Dasyprocta spp) indicate that, as was reported for other species, callosal connections are not limited to the midline segments of the body, but also include the representation of the digits of the forepaw (A. Pereira Jr., unpublished results).

As discussed in this review, evidence from the application of these techniques suggests that, at least in the visual system, the corpus callosum operates as a predictionenabling device by selecting the features to be processed. Further studies will determine whether the proposed anticipatory mechanism could be extended to other sensory modalities, and even represent a general principle of interhemispheric cooperation, also applicable to associative cortices.

\section{Acknowledgments}

The authors are grateful to Adiel B. Nascimento, Beth C.P. Moraes, and Antonia L. Carvalho for their collaboration, to Danilo Furtado for insightful discussions, and to Prof. Suzana Herculano-Houzel for revising the manuscript.

\section{References}

1. Kennedy H, Meissirel C \& Dehay C (1991). Callosal pathways and their compliancy to general rules governing the organization of corticocortical connectivity. In: Dreher $\mathrm{B} \&$ Robinson SR (Editors), Vision and Visual Dysfunction. Vol. 3. Neuroanatomy of the Visual Pathways and their Development. McMillan Press, London, UK, 324359.

2. Stryker MP \& Antonini A (2001). Factors shaping the corpus callosum. Journal of Comparative Neurology, 433: 437-440.

3. Berlucchi G (1981). Recent advances in the analysis of the neural substrate of interhemispheric communication. In: Pompeiano O \& Ajmone Marsan C (Editors), Brain Mechanisms and Perceptual Awareness. Raven Press, New York, NY, USA, 133-152.

4. Ramón y Cajal S (1898). Estructura del quiasmo óptico y teoria general de los entrecruzamientos nerviosos. Revista Trimestral Micrográfica, 3: 2-18.

5. Houzel JC \& Milleret C (1999). Visual in- ter-hemispheric processing: constraints and potentialities set by axonal morphology. Journal of Physiology (Paris), 93: 271284.

6. Innocenti GM (1986). General organization of callosal connection in the cerebral cortex. In: Jones EG \& Peters A (Editors), Cerebral Cortex. Vol. 5. Plenum Press, New York, NY, USA, 291-353.

7. Payne BR (1994). Neuronal interactions in cat visual cortex mediated by the corpus callosum. Behavioural Brain Research, 64: 55-64.

8. Hubel DH \& Wiesel TN (1967). Cortical and callosal connections concerned with the vertical meridian of the visual field in the cat. Journal of Neurophysiology, 30: 1561-1573

9. Berlucchi G \& Rizzolatti G (1968). Binocularly driven neurons in visual cortex of split chiasm cats. Science, 159: 308-310.

10. Milleret C, Houzel JC \& Buser P (1994). Pattern of development of the callosal transfer of visual information to cortical areas 17 and 18 in the cat. European Journal of Neuroscience, 6: 193-202.

11. Boyd JD \& Matsubara JA (1994). Tangential organization of callosal connectivity in the cat's visual cortex. Journal of Comparative Neurology, 347: 197-210.

12. Olavarria JF \& Abel PL (1996). The distribution of callosal connections correlates with the pattern of cytochrome oxidase stripes in visual area $\mathrm{V} 2$ of macaque monkey. Cerebral Cortex, 6: 631-639.

13. Sinich LC \& Horton JC (2002). Divided by cytochrome oxidase: a map of the projection from V1 to V2 in macaques. Science, 295: 1734-1737.

14. Schmidt KE, Kim DS, Singer W, Bonhoeffer T \& Löwel S (1997). Functional specificity of long-range intrinsic and interhemispheric connections in the visual cortex of strabismic cats. Journal of Neuroscience, 17: 5480-5492.

15. Milleret C \& Houzel JC (2001). Visual interhemispheric transfer to areas 17 and 18 in cats with convergent strabismus. 
European Journal of Neuroscience, 13: 137-152.

16. Innocenti GM \& Frost DO (1979). Effects of visual experience on the maturation of the efferent system of the corpus callosum. Nature, 280: 231-234.

17. Bosking $\mathrm{WH}$, Kretz $\mathrm{R}$, Pucak $\mathrm{ML}$ \& Fitzpatrick D (2000). Functional specificity of callosal connections in tree shrew striate cortex. Journal of Neuroscience, 20: 2346-2359.

18. Bosking $W H$, Zhang $Y$, Schofield $B$ \& Fitzpatrick D (1997). Orientation selectivity and the arrangement of horizontal connections in tree shrew striate cortex. Journal of Neuroscience, 17: 2112-2127.

19. Olavarria JF (1996). Non-mirror symmetric patterns of callosal linkages in areas 17 and 18 in cat visual cortex. Journal of Comparative Neurology, 366: 643-655.

20. Olavarria JF (2001). Callosal connections correlate preferentially with ipsilateral cortical domains in cat areas 17 and 18, and with contralateral domains in the $17 / 18$ transition zone. Journal of Comparative Neurology, 433: 441-457.

21. Alexeenko SV, Toporova SN \& Makarov FN (2001). The fine structure of interhemispheric connections in areas 17,18 of the cat. Perception, 30 (Suppl): 115 (Abstract).

22. Houzel JC, Milleret C \& Innocenti GM (1994). Morphology of callosal axons interconnecting areas 17 and 18 of the cat. European Journal of Neuroscience, 6: 898-917.

23. Swindale NV, Shoham D, Grinvald A, Bonhoeffer T \& Hübener M (2000). Visual cortex maps are optimized for uniform coverage. Nature Neuroscience, 3: 822826.

24. Payne BR (1991). Visual field map in the transcallosal sending zone of area 17 in the cat. Visual Neuroscience, 7: 201-219.

25. Payne BR \& Siwek DF (1991). Visual field map in the callosal recipient zone at the border between areas 17 and 18 in the cat. Visual Neuroscience, 7: 221-236.

26. Sanides D \& Albus K (1980). The distribution of interhemispheric projections in area 18 of the cat: coincidence with discontinuities of the representation of the visual field in the second visual area (V2). Experimental Brain Research, 38: 237240.

27. Harvey AR (1980). A physiological analysis of subcortical and commissural projections of areas 17 and 18 of the cat. Journal of Physiology, 302: 507-534.

28. McCourt ME, Thalluri G \& Henry GH (1990). Properties of area $17 / 18$ border neurons contributing to the visual trans- callosal pathway in the cat. Visual Neuroscience, 5: 83-98.

29. Czeiger D \& White EL (1993). Synapses of extrinsic and intrinsic origin made by callosal projection neurons in mouse visual cortex. Journal of Comparative Neurology, 330: 502-513.

30. Sun JS, Li B, Ma MH \& Diao YC (1994). Transcallosal circuitry revealed by blocking and disinhibiting callosal input in the cat. Visual Neuroscience, 11: 189-197.

31. Arieli A, Sterkin A, Grinvald A \& Aertsen A (1996). Dynamics of ongoing activity: explanation of the large variability in evoked cortical responses. Science, 273: 18681871.

32. Tsodyks $M$, Kenet $T$, Grinvald $A$ \& Arieli $A$ (1999). Linking spontaneous activity of single cortical neurons and the underlying functional architecture. Science, 286: 1943-1946.

33. Fries $P$, Neuenschwander $S$, Engel AK, Goebel R \& Singer W (2001). Rapid feature selective neuronal synchronization through correlated latency shifting. Nature Neuroscience, 4: 194-200.

34. Singer W (1999). Neuronal synchrony: a versatile code for the definition of relations? Neuron, 24: 49-65.

35. Lestienne R (2001). Spike timing, synchronization and information processing on the sensory side of the central nervous system. Progress in Neurobiology, 65: 545-591.

36. Zufferey PD, Jin F, Nakamura $H$, Tettoni $L$ \& Innocenti GM (1999). The role of pattern vision in the development of corticocortical connections. European Journal of Neuroscience, 11: 2669-2688.

37. Singer W (1995). Development and plasticity of cortical processing architectures. Science, 270: 758-764.

38. Schmidt KE, Goebel R, Löwel S \& Singer $W$ (1997). The perceptual grouping criterion of colinearity is reflected by anisotropies of connections in the primary visual cortex. European Journal of Neuroscience, 9: 1083-1089.

39. Innocenti GM, Lehmann P \& Houzel JC (1994). Computational structure of visual callosal axons. European Journal of Neuroscience, 6: 918-935.

40. Thorpe S, Fize D \& Marlot C (1996). Speed of processing in the human visual system. Nature, 381: 520-522.

41. Watroba L, Milleret C \& Buser P (2001). Impairment of binocular vision in the adult cat induces plastic changes in the callosal cortical map. European Journal of Neuroscience, 14: 1021-1029.

42. Das A \& Gilbert CG (1999). Topography of contextual modulations mediated by short-range interactions in primary visual cortex. Nature, 399: 655-661.

43. Olavarria JF \& Van Sluyters RC (1983). Widespread callosal connections in infragranular visual cortex of the rat. Brain Research, 279: 233-237.

44. Lewis JW \& Olavarria JF (1995). Two rules for callosal connectivity in striate cortex of the rat. Journal of Comparative Neurology, 361: 119-137.

45. Lappe M \& Hoffmann KP (2000). Optic flow and eye movements. International Review of Neurobiology, 44: 29-47.

46. Corballis MC \& Beale IL (1970) Bilateral symmetry and behavior. Psychological Review, 77: 451-464.

47. Segraves MA \& Rosenquist AC (1982). The afferent and efferent callosal connections of retinotopically defined areas in cat cortex. Journal of Neuroscience, 2: 1090-1107.

48. Spatz WB \& Tigges J (1972). Experimental anatomical studies on the "middle temporal visual area (MT)" in primates. I. Efferent cortico-cortical connections in the marmoset Callithrix jacchus. Journal of Comparative Neurology, 146: 451-464.

49. Orihara YI, Kishikawa M \& Ono K (1997). The fates of the callosal neurons in neocortex after bisection of the corpus callosum, using the technique of retrograde labeling with two fluorescent dyes. Brain Research, 778: 393-396.

50. Segraves MA \& Innocenti GM (1985). Comparison of the distribution of ipsilaterally and contralaterally projecting corticocortical neurons in cat visual cortex using two fluorescent tracers. Journal of Neuroscience, 5: 2107-2118.

51. Innocenti GM, Frost DO \& Illes J (1986). Maturation of visual callosal connections in visually deprived kittens: a challenging critical period. Journal of Neuroscience, 5: 255-267.

52. Innocenti GM (1980). The primary visual pathway through the corpus callosum: morphological and functional aspects in the cat. Archives Italiennes de Biologie, 118: 124-188.

53. Engel $A K$, König $P$, Kreiter $A K \&$ Singer $W$ (1991). Interhemispheric synchronization of oscillatory neuronal responses in cat visual cortex. Science, 252: 1177-1179.

54. Munk MHJ, Nowak LG, Nelson JI \& Bullier J (1995). Structural basis of cortical synchronization. 2. Effects of cortical lesions. Journal of Neurophysiology, 74: 2401-2414.

55. Salin PA \& Bullier J (1995). Corticocortical connections in the visual system: struc- 
ture and function. Physiological Reviews, 75: 107-154.

56. Kiper DC, Knyazeva MG, Tettoni L \& Innocenti GM (1999). Visual stimulusdependent changes in interhemispheric EEG coherence in ferrets. Journal of Neurophysiology, 82: 3082-3094.

57. Manger PR, Kiper D, Masiello I, Murillo L, Tettoni L, Hunyadi Z \& Innocenti GM (2002). The representation of the visual field in three extrastriate areas of the fer- ret (Mustela putorius) and the relationship of retinotopy and field boundaries to callosal connectivity. Cerebral Cortex, 12: 423-437.

58. White LE, Bosking WH, Williams SM \& Fitzpatrick D (1999). Maps of central visual space in ferret V1 and V2 lack matching input from the two eyes. Journal of Neuroscience, 19: 7089-7099.

59. Martinich S, Pontes MN \& Rocha-Miranda CE (2000). Patterns of corticocortical, corticotectal and commissural connections in the opossum visual cortex. Journal of Comparative Neurology, 416: 224244.

60. Shuler MG, Krupa DJ \& Nicolelis MAL (2001). Bilateral integration of whisker information in the primary somatosensory cortex of rats. Journal of Neuroscience, 21: $5251-5261$ 